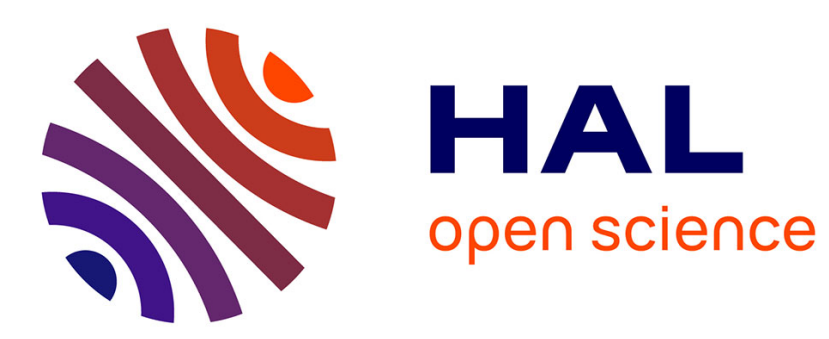

\title{
Local probe in a Rayleigh-Benard experiment in liquid helium
}

\author{
A. Libchaber, J. Maurer
}

\section{To cite this version:}

A. Libchaber, J. Maurer. Local probe in a Rayleigh-Benard experiment in liquid helium. Journal de Physique Lettres, 1978, 39 (21), pp.369-372. 10.1051/jphyslet:019780039021036900 . jpa-00231519

\section{HAL Id: jpa-00231519 https://hal.science/jpa-00231519}

Submitted on 1 Jan 1978

HAL is a multi-disciplinary open access archive for the deposit and dissemination of scientific research documents, whether they are published or not. The documents may come from teaching and research institutions in France or abroad, or from public or private research centers.
L'archive ouverte pluridisciplinaire HAL, est destinée au dépôt et à la diffusion de documents scientifiques de niveau recherche, publiés ou non, émanant des établissements d'enseignement et de recherche français ou étrangers, des laboratoires publics ou privés. 


\title{
LOCAL PROBE IN A RAYLEIGH-BENARD EXPERIMENT IN LIQUID HELIUM
}

\author{
A. LIBCHABER and J. MAURER \\ Groupe de Physique des Solides $\left({ }^{*}\right)$ de l'Ecole Normale Supérieure, \\ 24, rue Lhomond, 75231 Paris Cedex 05, France
}

(Reçu le 22 juin 1978, accepté le 19 septembre 1978)

\begin{abstract}
Résumé. - Sur une expérience de Rayleigh-Benard dans l'hélium liquide, nous avons étudié les effets dépendants du temps au-dessus du seuil de convection. Les résultats dépendent fortement du facteur de forme $\Gamma$, rapport du rayon de la cellule cylindrique à sa hauteur. Pour $\Gamma=2$ et $\Gamma=2,5$, nous observons des oscillations bien définies pour une grande gamme de nombres de Rayleigh. Pour $\Gamma=6$, un spectre de bruit basse fréquence apparaît à $R / R_{\mathrm{c}}=2$. Pour $R / R_{\mathrm{c}}>3,5$, le spectre de bruit présente un maximum local autour de $\omega \sim 14$. ( $\omega$ fréquence sans dimension, $R_{\mathrm{c}}$ nombre de Rayleigh critique d'établissement de la convection.) Cet effet persiste pour $\Gamma=4$ et $\Gamma=12$. $\Gamma=3$ représente donc le point de transition entre ces deux régimes.
\end{abstract}

\begin{abstract}
We have measured, in a Rayleigh-Benard experiment in liquid helium, the time dependent effects occurring above the onset of convection, using a local probe. Results are very dependent on the aspect ratio $\Gamma$, ratio of the cylinder cell radius to its height. For a small aspect ratio, $\Gamma=2$ and $\Gamma=2.5$, well defined sharp oscillations are present for a large range of Rayleigh numbers. For $\Gamma=6$, a low frequency noise appears for $R / R_{\mathrm{c}}=2$, where $R_{\mathrm{c}}$ is the critical Rayleigh number for convection. No well-defined oscillations are present but the noise peaks at a dimensionless frequency around $\omega=14$, when $R / R_{\mathrm{c}}>3.5$. This effect exists also for $\Gamma=4$ and $\Gamma=12 . \Gamma=3$ is thus a transition point between the two regimes.
\end{abstract}

We report here some preliminary new results on a Rayleigh-Benard experiment in liquid helium, using for the first time a local probe. Time dependent effects above the convection threshold are the main focus of this experiment.

The transitions to turbulent convection have been extensively studied recently $[1,2]$. Of particular relevance to this paper is the work of G. Ahlers and R. P. Behringer $[3,4,5]$ on ${ }^{4} \mathrm{He}$ above the lambda transition. It is a detailed study, in a RayleighBenard experiment, of the various transitions occurring for the effective thermal conductivity of ${ }^{4} \mathrm{He}$, as one heats from below a sample of the liquid placed between two plates. The experimental advantages of low temperature techniques have been clearly demonstrated [5], i.e., thermal measurements of very high resolution and great accuracy. Up to now the two important limitations of a low temperature experiment in ${ }^{4} \mathrm{He}$ are, first, the difficulty of direct observation

$\left(^{*}\right)$ Laboratoire associé au Centre National de la Recherche Scientifique. of the convective roll pattern, and second the absence of local probes. We wish to report here the results obtained, using a local probe, on the time-dependent temperature fluctuations which present a completely different character, as one goes from a small aspect ratio $\Gamma=2$, to a large one $\Gamma=12(\Gamma=D / 2 d$, $D$ cell diameter, $d$ cell height).

1. Experimental situation. - The experimental cell is shown on figure 1 . It is a cylinder with a variable height and a diameter $D=25 \mathrm{~mm}$. The top and bottom boundaries are made out of copper. The wall consists of a $0.2 \mathrm{~mm}$ thick stainless steel cylinder. To change the aspect ratio $\Gamma$, we introduce teflon gaskets which change the diameter $D$. The cell is surrounded by a very high vacuum, better than $10^{-6}$ torr. Thermal regulation for the top plate provides a temperature stability regulation of the order of $10^{-6} \mathrm{~K}$. To reach such a stability, we increase the heat capacity of the temperature regulated ensemble by adding a second reservoir of helium, in series with the experimental cell and regulated by the same temperature control. 


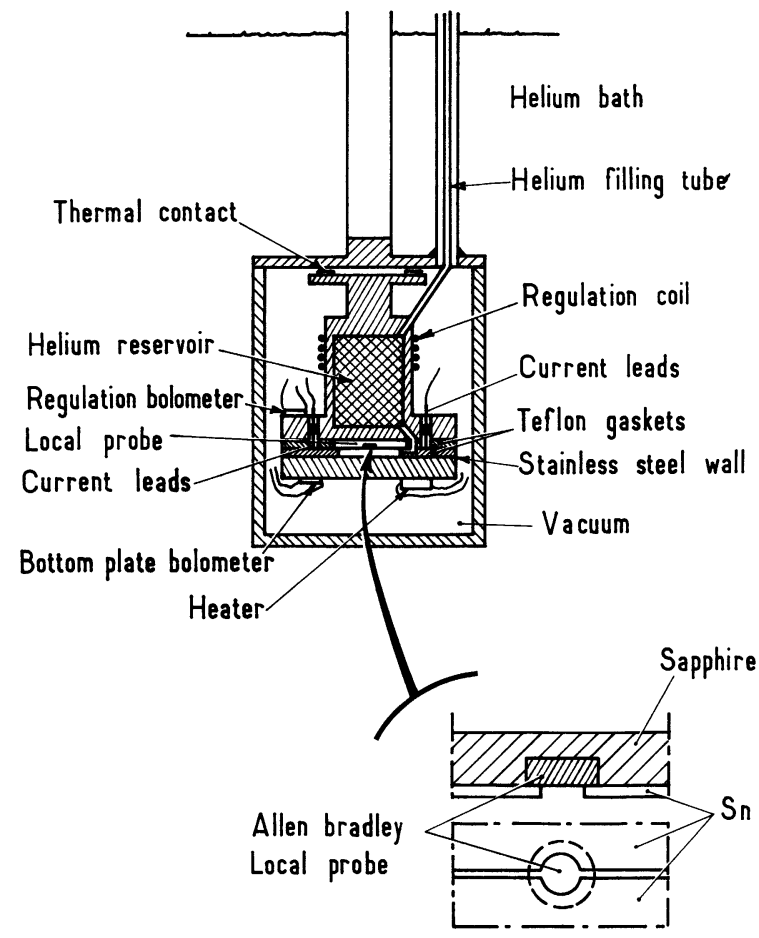

FIG. 1. - The upper part is the detail of the experimental system. The lower part is an enlarged view of the local probe seen both in a transverse cut and from the bottom. Dimensions are not conserved.

This reservoir is packed with porous bronze. Another advantage of this reservoir is that it avoids the thermal problems introduced by the helium column required to fill up the experimental chamber. The fluid properties are obtained from the NBS chart [6]. The various temperatures are measured, using Allen Bradley resistors. The bolometers are thinned down to $0.3 \mathrm{~mm}$ and glued with a General Electric varnish to the upper and bottom plates.

2. The local probe. - A local probe is placed in the upper plate, in the following manner (see the enlargement on Fig. 1). A sapphire plate of thickness $1.3 \mathrm{~mm}$, glued to the top copper boundary of the cell is the support of the local probe. A small hole (diameter $0.8 \mathrm{~mm}$, depth $0.4 \mathrm{~mm}$ ) is drilled by ultrasound technique in the centre of the sapphire, being thus located on the revolution axis of the cell. A small carbon disc, taken out from an Allen Bradley resistor, fills the hole and is glued to it using stycast. The sapphire plate is then lapped with an abrasive powder for flatness, the carbon element being flush with the surface. About one micrometer of tin is then evaporated and covers the whole plate. Using a micromanipulator, an area of the carbon disc is uncovered, thus taking off the tin layer, and various cuts on the tin thin film are machined to insure the electrical connections to the bolometer. The final resistance of the bolometer at $4.02 \mathrm{~K}$ is about $30000 \Omega$. This local probe detects temperature changes on an area of about 0.2 square millimeter.

A small amplitude low frequency a.c. signal is applied to the bolometer $\left(80 \mathrm{~Hz}, 3 \times 10^{-9} \mathrm{~W}\right)$. The measurements are performed using a lock-in detector which is placed after a balanced bridge. The bridge is at helium temperature and the local probe constitutes one arm of the bridge. The signal is then Fourier analysed using a 5420 A Hewlett Packard Digital signal analyser. Typically a spectrum is averaged a few hundred times using a sliding average program.

The bolometer noise has a $1 / f$ overall variation as shown on figure 4 , in the whole frequency domain of study, which ranges from $1 \mathrm{mHz}$ to $5 \mathrm{~Hz}$. The fluid parameters and cell-dimensions are given in table $\mathbf{I}$.

3. Experiment. - In a Rayleigh-Benard experiment, the two dimensionless numbers of interest are the Rayleigh number $R=g \alpha d^{3} \Delta T / K v$ and the Prandtl number $\sigma=v / K$ where $g$ is the acceleration of gravity, $\alpha$ the isobaric thermal expansion coefficient, positive in our case, $K$ the thermal diffusivity and $v$ the kinematic viscosity. The variable is the temperature difference between the two plates $\Delta T$, and the onset of convection is for $R=R_{\mathrm{c}}$ where $R_{\mathrm{c}}=1708$ for large $\Gamma$ [7]. The Prandtl number measures the relative importance of vorticity diffusion to heat conduction. In our case, where $\sigma \sim 0.6$, heat diffusion and vorticity diffusion are of the same order. The convection threshold $R_{\mathrm{c}}$ is obtained from a heat conductivity measurement.

In our measurement of the time-dependent fluid flow, we impose the heat current $\dot{q}$ and measure the temperature difference $\Delta T$. The heat flows in parallel through the teflon boundaries and helium. Our measurements are very dependent on the aspect ratio, we will thus present them for increasing aspect ratio.

.4. Small aspect ratio. -4.1 ASPECT RATIO $\Gamma=2.5$. - For $\Gamma=2.5$, very well defined time dependent oscillations are recorded by the local

TABLE I

Fluid properties and cell dimension

\begin{tabular}{|c|c|c|c|c|c|c|c|c|c|}
\hline $\begin{array}{c}\mathrm{cm} \\
D\end{array}$ & & $\mathrm{~cm}$ & $\begin{array}{l}\mathrm{K} \\
T\end{array}$ & $\underset{P}{\text { Atm }}$ & $\begin{array}{l}\text { Poise } \\
10^{6} n\end{array}$ & $\begin{array}{l}\mathrm{cm}^{2} / \mathrm{s} \\
10^{4} K\end{array}$ & $\begin{array}{l}\mathrm{cm}^{2} / \mathrm{s} \\
10^{4} v\end{array}$ & $\sigma$ & $\begin{array}{c}\mathrm{s} \\
d^{2} / K\end{array}$ \\
\hline- & & - & - & - & - & - & - & - & - \\
\hline $\begin{array}{c}\Gamma=2 \\
4 \times 10^{-1}\end{array}$ & $\begin{array}{c}\Gamma=6 \\
1.2\end{array}$ & $10^{-1}$ & 4.022 & 2.5 & 37 & 4.3 & 2.7 & 0.63 & 23.2 \\
\hline
\end{tabular}


probe, as shown on figure 2 . We observe sharp spectral lines with a large number of harmonics, up to the tenth order. They start for $R / R_{\mathrm{c}}$ slightly larger than $2\left(R_{\mathrm{c}}\right.$ is the critical Rayleigh number for convection).

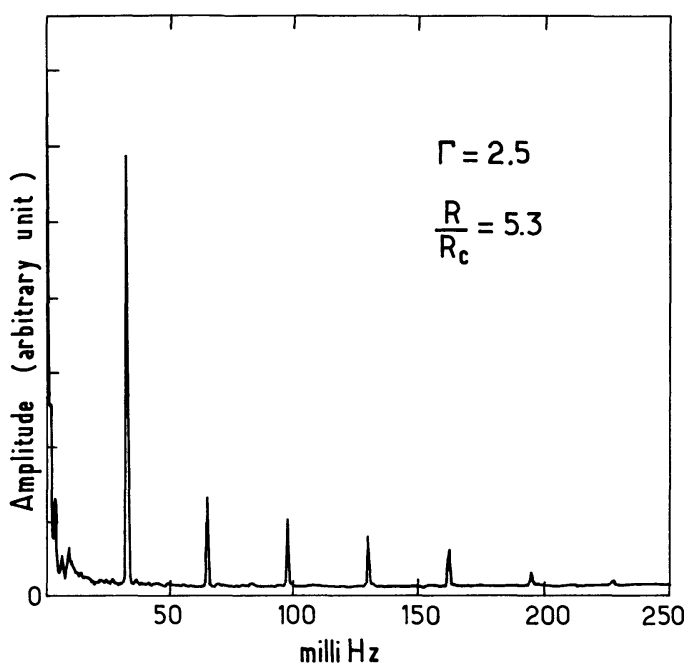

FIG. 2. - Recordings of the Fourier frequency analysis for $\Gamma=2.5$ and $\frac{R}{R_{\mathrm{c}}}=5.3$. The amplitude and the frequency are on a linear scale. The peak frequency is $32.4 \mathrm{mHz}$ with six harmonics.

The measured frequency, in the non-dimensional unit $\omega=2 \pi \frac{d^{2}}{K} f$, is $\omega=1.45$. The evolution of the spectra is shown on figure 3 , the data corresponding to the circles. The frequency changes abruptly for $\frac{R}{R_{\mathrm{c}}} \sim 4.7$ from $\omega \sim 2$ to $\omega \sim 4$. At $\frac{R}{R_{\mathrm{c}}} \sim 6$, another abrupt change appears for $\omega \sim 5$ to $\omega \sim 8.5$ and a

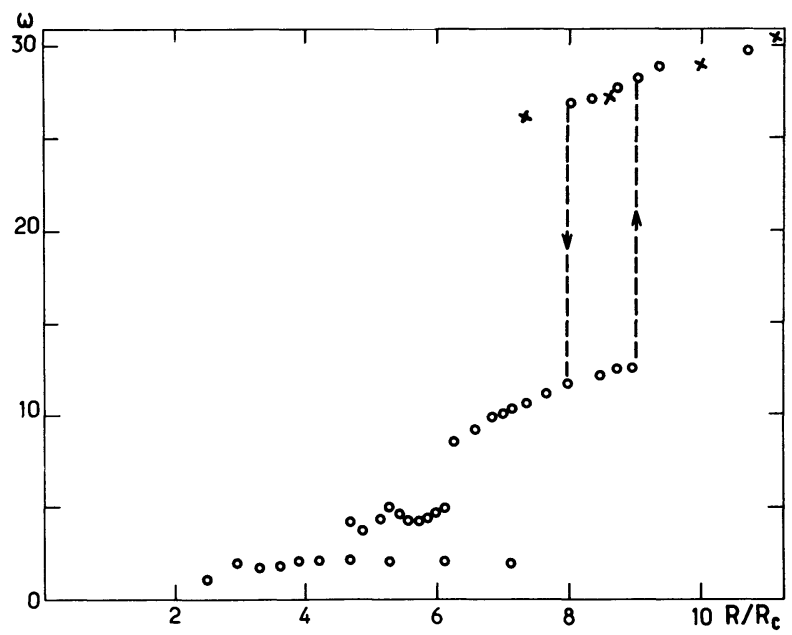

FIG. 3. - Plot of the observed frequencies as a function of $\frac{R}{R_{\mathrm{c}}}$ for $\Gamma=2.5$ and $\Gamma=2$. The frequencies are normalized in the dimensionless unit $\omega=\overline{\left(2 \pi \frac{d^{2}}{K}\right)} f$. The dotted line shows the hysteresis curve. Data for $\Gamma=2$ correspond to the crosses. Data for $\Gamma=2.5$ correspond to the circles. final one at $\frac{R}{R_{\mathrm{c}}} \sim 8$ from $\omega \sim 12.5$ to $\omega \sim 27$. For $\frac{R}{R_{\mathrm{c}}} \sim 12$ a low frequency continuous noise spectrum appears, and slowly spreads over the entire spectrum, as one increases the Rayleigh number. For $\frac{R}{R_{\mathrm{c}}} \sim 25$ the peaks are fully covered by noise. In each case, the frequency seems to increase by a factor of two within $20 \%$. Also for several values of $R / R_{\mathrm{c}}$ and especially at each transition the original frequency $\omega \sim 2$ reappears.

Thus for $\frac{R}{R_{\mathrm{c}}}>4.5$, two frequencies seem to coexist and their values are in reasonable agreement with Ahlers and Behringer [3] results ; to be more explicit, the lower frequency is not always present for all values of $R / R_{\mathrm{c}}$ but reappears for some values of $R / R_{\mathrm{c}}$ and these are shown on figure 3 . But the quasiperiodic state starts in our case at $\frac{R}{R_{\mathrm{c}}} \sim 2$, which is different from Ahlers observation where time periodic flow starts at $\frac{R}{R_{\mathrm{c}}} \sim 10$. Also, the high frequency oscillation seems to originate from the low frequency one, as a type of cell division. Finally, as can be seen in figure 3 , an hysteresis loop is present for the last transition.

4.2 Aspect ratio $\Gamma=2$. - The data are similar to those for the previous case, as shown on figure 3 by the crosses, with one main difference : we observe only one branch of the oscillation spectrum and it starts at $\frac{R}{R_{\mathrm{c}}}=7$.

5. Large aspect ratio $\Gamma=6$. - The behaviour is totally different. We do not observe any temperature oscillations with well defined frequencies. Typical curves are shown on figure 4 for $\Gamma=6$. Measuring the power spectral density, we detect the start of very low frequency noise for $\frac{R}{R_{\mathrm{c}}} \sim 2$, which follows closely Ahlers' analysis. The evolution of the noise spectrum is the following : it starts at $\frac{R}{R_{\mathrm{c}}} \sim 2$, as a very low frequency noise with a maximum at $\omega=0$. As one increases the Rayleigh number, noise spreads to higher frequencies and when it reaches a frequency of about $10^{-1} \mathrm{~Hz}(\omega=14)$, a broad peak appears, as shown on figure 4 for $\frac{R}{R_{\mathrm{c}}}=3.7$. The position of the peak slightly varies with $\frac{R}{R_{\mathrm{c}}}$ and a second harmonic is generated for $\frac{R}{R_{\mathrm{c}}}=4.5$. This broad peak is already present for an aspect ratio $\Gamma=4$ and still exists for $\Gamma=12$ with up to three harmonics. 


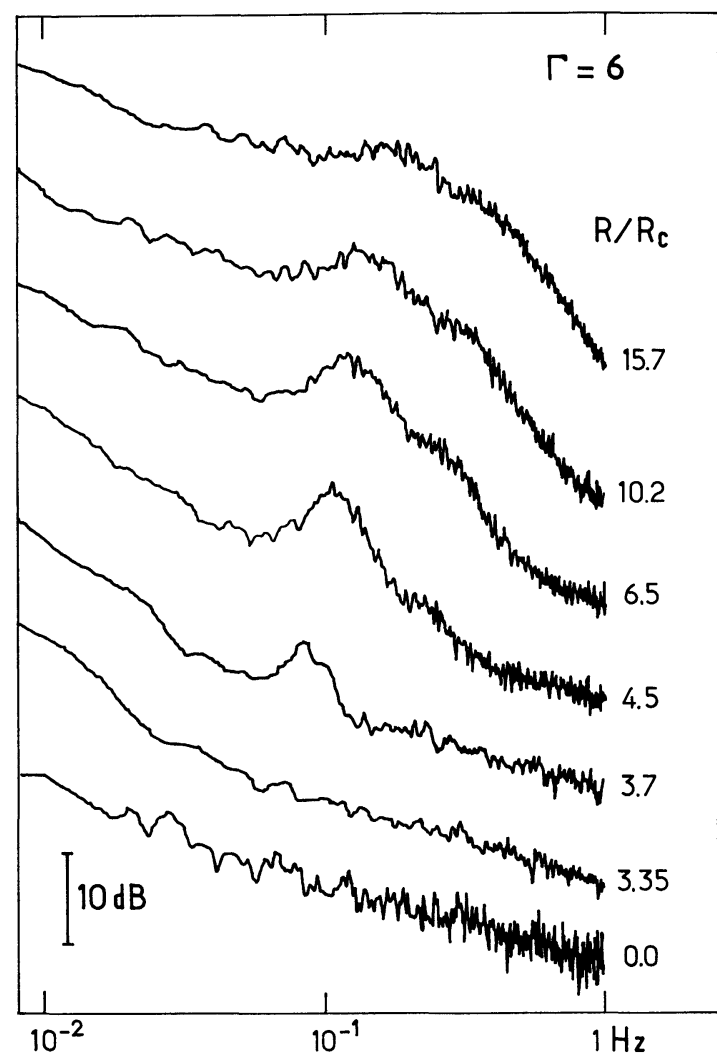

6. Conclusion. - Our main conclusion is the extraordinary dependence of the results on the aspect ratio. Whereas for $\Gamma=2$ and $\Gamma=2.5$ well resolved time dependent oscillations exist, for $\Gamma=4,6$, a low frequency noise sets in around $\frac{R}{R_{\mathrm{c}}} \sim 2$ and a broad peak centred around $\omega=14$ appears for $\frac{R}{R_{\mathrm{c}}} \simeq 4$. The aspect ratio $\Gamma=3$ is thus the transition between a regime with well defined oscillations for $\Gamma<3$ to a regime with a low îrequency noise and a broad peak structure starting at $\frac{R}{R_{\mathrm{c}}} \sim 2$ for $\Gamma>3$. Before presenting a theoretical analysis of the data, we are now performing experiments with two probes, studying the spatial correlation. Nevertheless one can suggest that the transition observed may be from a set of concentric rolls to a geometry where the rolls are perpendicular to the lateral boundary.

FIG. 4. - Power spectra as a function of frequency for $\Gamma=6$. Both coordinates are in logarithmic scale; the curves have been translated on the vertical axis for clarity. The lower curve shows the $1 / f$ character of the local bolometer.

\section{References}

[1] Krishnamurti, R., J. Fluid Mech. 60 (1973) 285.

[2] Normand, C., Pomeau, Y., Velarde, M. G., Rev. Mod. Phys. 49 (1977) 581.

[3] Ahlers, G., Behringer, R. P., Phys. Rev. Lett. 40 (1978) 712.

[4] Behringer, R. P., Ahlers, G., Phys. Lett. 62A (1977) 329.
[5] Ahlers, G., Phys. Rev. Lett. 33 (1974) 1185.

[6] MC CarTy, R. D., Thermophysical physical properties of ${ }^{4} \mathrm{He}$, Nat Bur. Stand. Tech. Note no 631 (1972).

[7] Pellew, A., Southwell, R. V., Proc. R. Soc, London A 176 (1940) 312 